\title{
Correction to: Comparisons of percutaneous versus retroperitoneoscopic cryoablation for renal masses
}

\author{
Published online: 18 September 2018 \\ (c) Springer Nature B.V. 2018

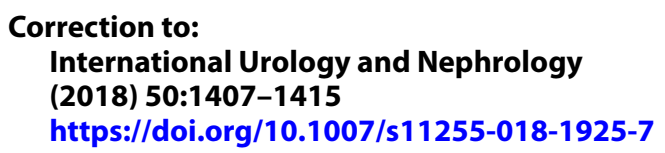

Hui-Ying Liu ${ }^{1} \cdot$ Shu-Huei Shen ${ }^{2,3} \cdot$ Lin-Nei Hsu $^{4} \cdot$ Po-Hui Chiang ${ }^{1}$

In the original publication, Prof. Lin-Nei Hsu's institution was incorrectly published as 'Tainan Municipal An-Nan Hospital' in affiliation 4. The correct affiliation should read as 'Department of Urology, An-Nan Hospital, China Medical University, Tainan, Taiwan'.

The original article can be found online at https://doi.org/10.1007/ s11255-018-1925-7.

Po-Hui Chiang

cphtem@yahoo.com.tw

Hui-Ying Liu

ying_1011@hotmail.com

Shu-Huei Shen

shshen@vghtpe.gov.tw

Lin-Nei Hsu

nei0217@gmail.com

1 Department of Urology, Kaohsiung Chang Gung Memorial Hospital, Chang Gung University College of Medicine, Kaohsiung, Taiwan

2 Department of Radiology, Taipei Veterans General Hospital, Taipei, Taiwan

3 School of Medicine, National Yang-Ming University, Taipei, Taiwan

4 Department of Urology, An-Nan Hospital, China Medical University, Tainan, Taiwan 